\title{
PROPERTIES OF NdDyFeCoTi MAGNETO-OPTICAL MEDIA MADE FROM CAST ALLOY TARGET
}

\author{
T. SHIMODA, S. SHIMOKAWATO, S. FUNADA, S. NEBASHI, A. AOYAMA, \\ and M. SUGIMOTO \\ Research and Development Division, SEIKO EPSON Corp., Suwa, Nagano 392, Japan
}

\begin{abstract}
We have developed a magneto-optical recording media having a high performance and good reliability using more abundant rare earth elements than $\mathrm{Tb}$. By using a Na-Dy-Fe-Co-Ti composition, the alloy target is easily formed by the casting method. As a result, the oxygen content restricted to under $400 \mathrm{ppm}$. That enables the media to have a high performance and it is easy for mass production. Each element of $\mathrm{Nd}, \mathrm{DY}, \mathrm{Fe}, \mathrm{Co}$ and $\mathrm{Ti}$ plays an essential role in magneto-optical properties, magnetic properties, reliability and realization of the cast alloy target.
\end{abstract}

\section{INTRODUCTION}

Thin films composed of a Tb are reliable rare earth transition metal alloy films for high density magneto-optical recording. Because they have an amorphous structure and a large perpendicular magnetic anisotropy as well as an adequate Curie temperature Tc, ideal magneto-optical media can be produced by using a $\mathrm{Tb}$ compound in the laboratory.[1] However, if one tries to produce a large number of these media at a mass production level at low cost, there are some problems to be solved. These problems are here listed.

(1) The amount of natural resources of $\mathrm{Tb}$ is very small. That nakes $\mathrm{Tb}$ metal very expensive. Additionally, most $\mathrm{Tb}$ is utilized in fluorescent materials. Therefore, there are only smali amounts of $\mathrm{Tb}$ left for use in producing magnetooptical recording media.

(2) This film of magneto-optical media is mainly produced by a sputtering method. In that method, an alloy target which contains all the elements needed for a film is desirable. However, TbFe or TbFeCo is too fragile to obtain an alloy target if it is made by casting. Accordingly, the powder metallurgical method is adopted to obtain such target. That results in a high oxygen content ( ) $1000 \mathrm{ppm}$ ) and involves complex production steps.

In order to solve the above problems we have been studying a more viable alloy system with the following conditions. Namely, it should be composed of more abundant rare earth elements than $\mathrm{Tb}$. This alloy system also should enable the alloy target to be manufactured by simple casting. The magneto-optical medium made from this alloy system should have better properties than that containing Tb. $[2,3]$

\section{EXPERIMENTAL}

Alloy targets composed of $\mathrm{Nd}-\mathrm{Fe}, \mathrm{Nd}-\mathrm{Dy}-\mathrm{Fe}$ and $\mathrm{Nd}-\mathrm{Dy}-\mathrm{Fe}-\mathrm{Co}-\mathrm{Ti}$ were melted and cast into ingots in an induction furnace. These ingots were formed into disk-shaped targets for sputter deposition. The diameter and thickness of these targets were $10.16 \mathrm{~cm}$ ( 4 inches) and $0.4 \mathrm{~cm}$, respectively. Usually the oxygen content was as much as $400 \mathrm{ppm}$. The MO layer or alloy film was deposited by dc sputtering. Before sputter deposition, the chamber was evacuated to less than $5 \times 10^{-7}$ Torr. Deposition rates ranged from 10 to $100 \mathrm{~nm} / \mathrm{min}$. In the experiment of the Nd-Dy-Fe-Co system, a Nd-Dy and Fe target were used. Co tips was laid on a $\mathrm{Fe}$ target to adjust the Co content. To investigate the effects of

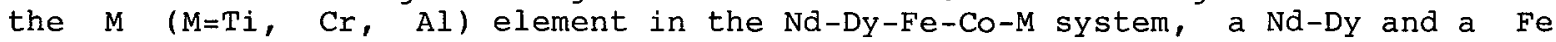
target were used and $M$ tips were also laid on Fe targets together with Co.

Glass substrates and polycarbonate disks were used for measuring magnetic properties and for evaluating read/write characteristics, respectively.

Magnetization(M), coercive force(HC) and Tc of the films were measured by a vibrating-sample magnetometer. The Kerr or Faraday rotation angle was measured at a wavelength of $780 \mathrm{~nm}$. An inductively coupled plasma analysis was used to determine the composition of the films. The amorphous structure of the film was confirmed by $\mathrm{X}$-ray and electron diffraction. 


\section{RFSULTS}

Generally, the light rare earth-iron system (LR-Fe) forms fewer intermetallic compounds than the heavy rare earth-iron system (HR-Fe). Therefore LR-Fe alloys have larger mechanical strength than HR-Fe alloys. The latter is very fragile. If magneto-optical recording media with good properties can be obtained by using the LR-Fe system, an alloy target for sputtering can be formed by simple casting. This is the starting point of our experiment.

First, we chose $\mathrm{Nd}$ as $\mathrm{LR}$ and made a $\mathrm{Nd}_{\mathbf{a}} \mathrm{Fe}_{100-a}$ alloy target to produce magnetic thin films. As a result, it was found that these films had an amorphous structure in the range of $a=20-40$. In the range of $a=15-30$, the anisotropy constant $K_{\perp}$, which is determined by the equation $K_{\perp}=\mathrm{Ku}-2 \pi M s^{2}$, was positive. The angle of Kerr rotation is about $15 \mathrm{~min}$ and $\mathrm{Tc}$ is $80{ }^{\circ} \mathrm{C}$ when $\mathrm{a}=30$. These values are relatively good, however, the Hc of the Nd-Fe thin film had a low value of 10 or 20 Oe. From this experiment we conclude that $\mathrm{Nd}$ can be used at least as one rare earth element in forming a magneto-optical recording medium by the $\mathrm{R}-\mathrm{Fe}$ system.

In order to obtain a higher Hc value while assuring that the film has good magneto-optical characteristics, and that an alloy target can be formed by casting, we investigated combination of Nd and heavy rare earth(HR). As the HR, we chose Dy because DY is a more abundant element and about ten times cheaper than $\mathrm{Tb}$. It is therefore not critical as a natural resource.

We studied the (NdxDy1-x)25Fe75 system and obtained the results shown in Fig.1. As $\mathrm{Ku}>2 \pi \mathrm{Ms}^{2}$ is the condition to get a perpendicular magnetized film, this system is worth studying for a magneto-optical medium in the range of $x<0.8$. However, $H c$ is still low even at $x=0.6$, where Hc is about 500 Oe. In order to realize a higher Hc than that in the above systems, the range of $x<0.5$ is investigated, so that $\mathrm{Hc}$ over $1 \mathrm{kOe}$ is feasible in the range of $x<0.6$. Hc reaches around 10 kOe at $x=0.3$ or 0.2 if the sputtering conditions are adequate. Next, we studied the effect of co using the composition of (Nd0.40 Dy0.60)30 (Fe1-xCox)70. In this system Co had no effect on He but it had a great effect on the Kerr rotation angle. This is shown in Fig.2. However, in other systems such as (Nd0.4 Dy0.6)35(Fe1-x Cox)65, an increase of Hc up to $x=0.3$ was observed. Of course, an increase in the Kerr rotation angle was also confirmed in this system.

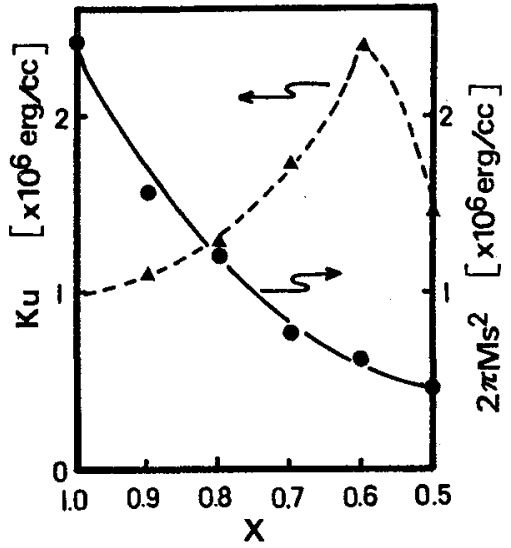

Fig. 1 Dependence of $M s(\bullet)$ and $\mathrm{Ku}(\mathbf{\Delta})$ on Nd content in Nd-DyFe amorphous alloy.

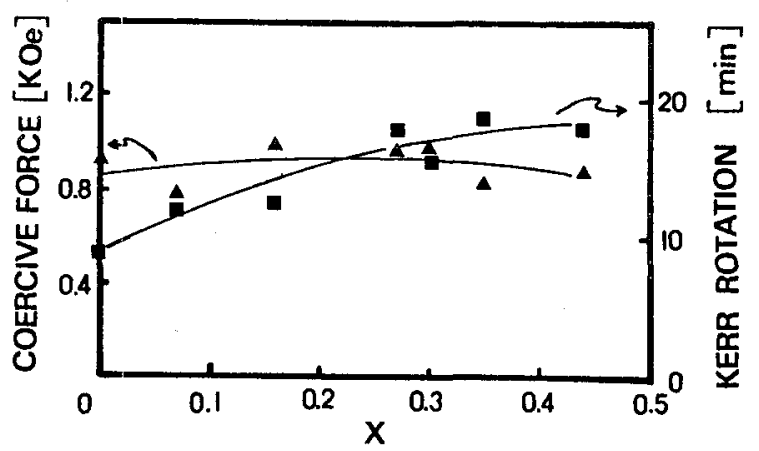

Fig.2 Dependence of Kerr rotation angle (E) and coercive force (A) on Co content in NaDy $(\mathrm{Fe} 1-\mathrm{xCOx})$.

As a result of these experiments and other similar ones, we have obtained an optimum composition range for a magneto-optical medium in the Nd-Dy-Fe-Co system. The typical composition is (Nd 0.2 Dy0.8)25(Fe0.7Co0.3)75.

In order to make the production of the alloy target easier by enhancing the mechanical strength of the cast alloy, M was added to the Nd-Dy-Fe-Co system. The special effects of Al, Cr or Ti were investigated, because these elements were reported to improve the reliability of the magneto-optical media in previous work[4]. If a certain element of those tested proved to have an important effect on making an alloy target simultaneously, it would be a significant breakthrough. As a result of serial work, we found that Ti had the greatest effect on improving the mechanical strength of an alloy while maintaining its magneto-optical and magnetic properties at a high level relative to the extent of $\mathrm{Ti}$ content. Table 2 shows magneto-optical and magnetic properties of the developed film containing $\mathrm{Ti}$ of 4 ats. This composition, (Nd0.23Dy0.77)25 (Fe0.55Co0.40Ti0.05)75 shown in Table 1, is the optimum composition in the $\mathrm{Nd}-\mathrm{Dy}-\mathrm{Fe}-\mathrm{Co}-\mathrm{Ti}$ alloy system. Using this composition, the alloy target is easily cast. 


\begin{tabular}{lrr}
\hline \multicolumn{1}{c}{ Properties } & & Value \\
\hline Magnetization & (emu/CC) & 40 \\
Coercive force & $(\mathrm{KO})$ & 8 \\
Curie temperature & $\left({ }^{\circ} \mathrm{C}\right)$ & 220 \\
Crystallization Temperature & $\left({ }^{\circ} \mathrm{C}\right)$ & 420 \\
Faraday Rotation angle & (deg/cm) & $2.0 \times 10^{5}$ \\
\hline
\end{tabular}

Table 1. Magnetic properties of (Nd0.23Dy0.77)25(Fe0.55Co0.40Ti0.05)75
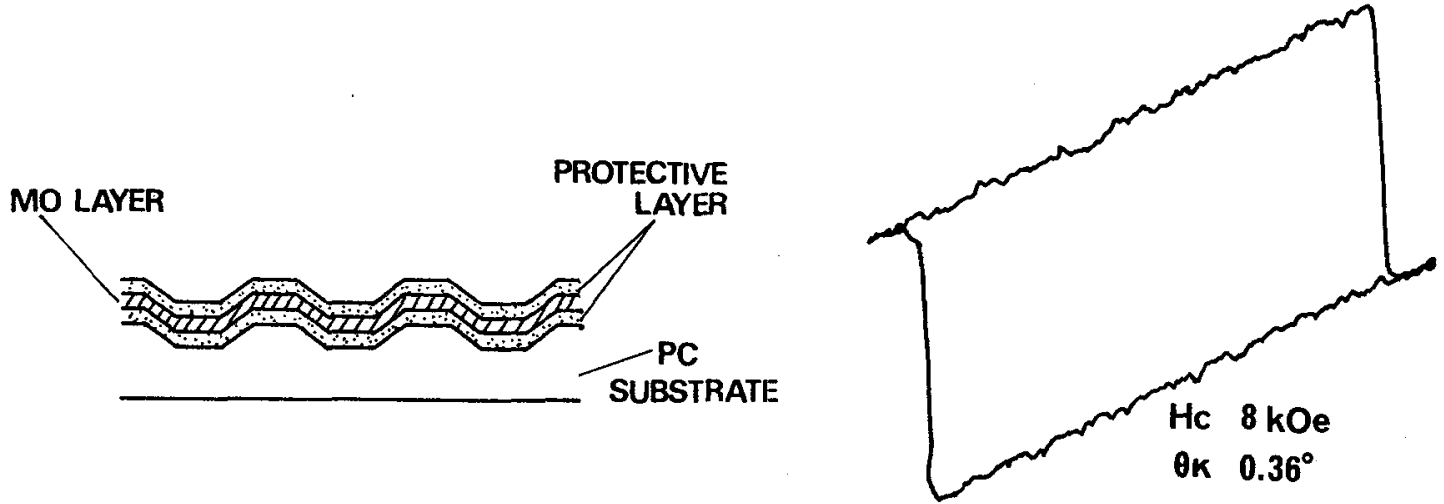

Fig. 3 structure of Mo media.

Fig.4 Kerr hysteresis loop of $\mathrm{NC}-\mathrm{DY}-\mathrm{Fe}-\mathrm{Co}-\mathrm{Ti}$ deposited on polycarbonate substrate.

We produced the magneto-optical (MO) media, in which the composition is the same as the one shown in Table 1, by sputtering an alloy target containing 5 elements. The structure of the media is shown in Fig.3. We used the polycarbonate substrate and inorganic materials as a protective layer of which the thickness is $100 \mathrm{~nm}$ and the refractive index is 2.0 . Fig. 4 shows the Kerr hysteresis loop of the fabricated media. The recording characteristics of this media were measured according to the conditions shown in Table 2. Fig.5 illustrates the relationship botween the carrier to noise ratio (CNR) and the magnetic field during recording. Fig.6 shows the dependence of CNR on the laser power during recording. An optimum CNR of over $50 \mathrm{~dB}$ was obtained at the recorded domain length of $1.7 \mu \mathrm{m}$ by using a recording layer of $100 \mathrm{~nm}$.

\begin{tabular}{lc}
\hline \multicolumn{1}{c}{ Contents } & Value \\
\hline Recording Power & $6.2 \mathrm{mw}$ \\
External Field & $200 \mathrm{Oe}$ \\
Carrier Frequency & $1.0 \mathrm{MHz}$ \\
Linear Velocity & $3.5 \mathrm{~m} / \mathrm{sec}$ \\
Duty Ratio & $50 \mathrm{~g}$ \\
Bit length & $1.7 \mu \mathrm{m}$ \\
Track pitch & $1.6 \mu \mathrm{m}$ \\
RBW & $30 \mathrm{kHz}$ \\
\hline
\end{tabular}

Table 1. Conditions for read/write characteristics evaluation.

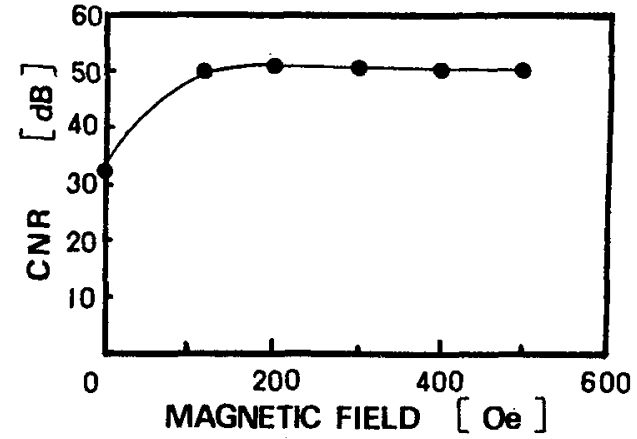

Fig. 5 Dependence of CNR on magnetic field.

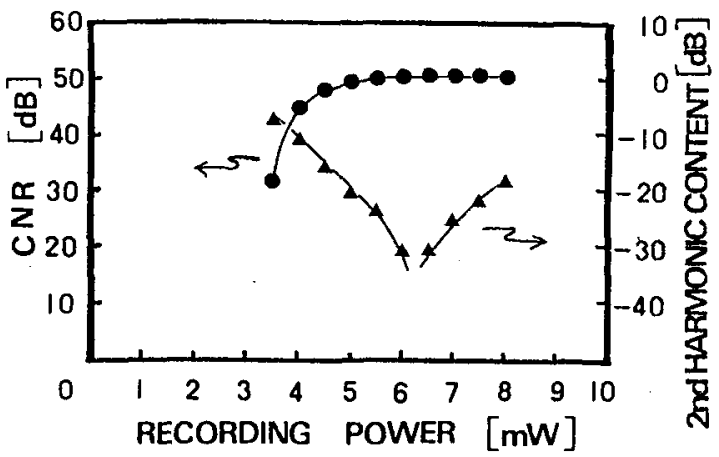

Fig. 6 Dependence of CNR on recording power.

Fig.7 illustrates the long term stability of the given media under the condition of $90 \% \mathrm{RH}$ at $70^{\circ} \mathrm{C}$. It remained stable after aging for over 1000 hours. 
In this diagram, critical times under certain conditions are shown, having been determined by Arrhenius's method.

We have found that Nd played an important role not only in forming the alloy target[2] but also in improving the recording characteristics of the magneto-optical medium.[3] Nd can also greatly improve the squareness of hysteresis near TC (Magnetic field vs. Kerr or Faraday rotation Angle), if Nd is added to the Dy-Fe-Co-Ti system. We believe this is a reason why the Dy-Fe-Co-Ti medium is improved by adding Nd. Fig. 8 shows the spectrum of readback signal from a MO medium having a composition of (Nd0.2Dy0.8)25(Fe0.58Co0.38Ti0.04)75. The writing noise coincides with the disk noise. That means that the above mentioned medium generates extremly low noise.

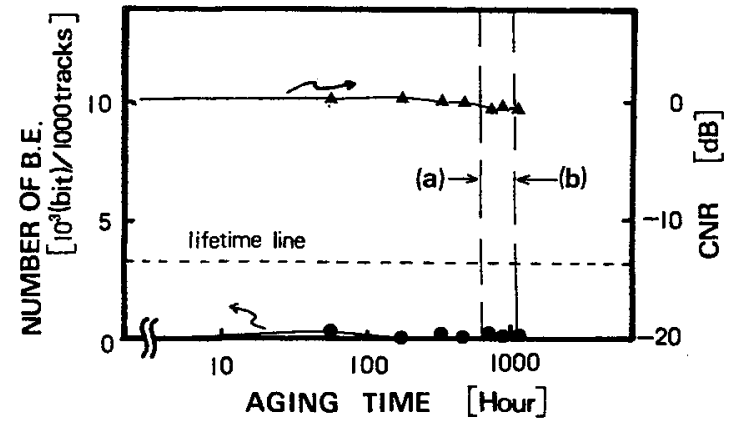

Fig.7 Change in number of bit error and carrier to noise ratio (CNR) as a function of aging time. The environment is maintained at $70{ }^{\circ} \mathrm{C} 908 \mathrm{RH}$. The lines (a) and (b) show a lifetime of ten years at $25^{\circ} \mathrm{C} 90 \% \mathrm{RH}$ and $30^{\circ} \mathrm{C} 90 \% \mathrm{RH}$, respectively.

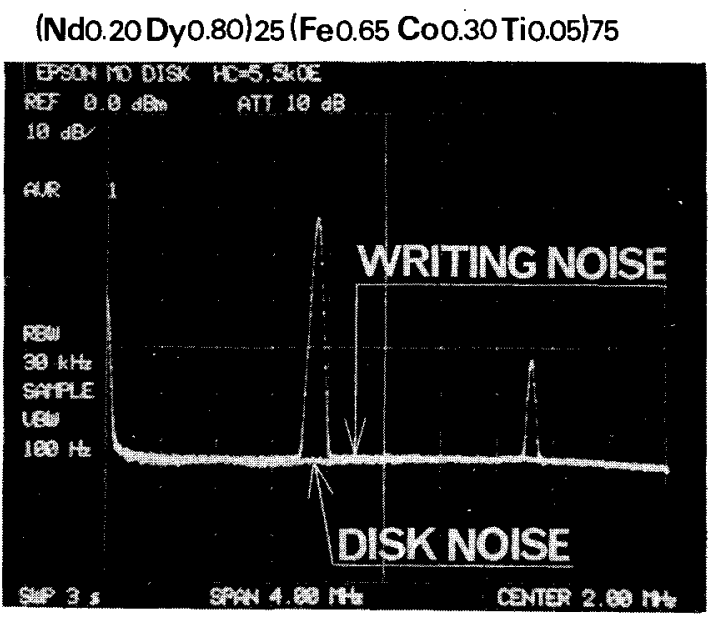

Fig.8 Spectrum of readback signal of NdDyFeCoTi

\section{SUMMARY}

(1) We have developed a magneto-optical recording media having both high performance and good stability using rare earth elements which are more abundant than $\mathrm{Tb}$.

(2) By using a Na-Dy-Fe-Co-Ti composition, an alloy target is easily formed through a casting method. As a result, oxygen content restricted to under $400 \mathrm{ppm}$, which enables the media to have both a high performance and the ability to be easily produced through mass production.

(3) $\mathrm{Nd}$ and $\mathrm{Ti}$ play important roles in the realization of the cast alloy. $\mathrm{Ti}$ is also supposed to contribute towards improving the reliability of the medium. Nd has also been found to improve the recording characteristics of the medium.

\section{ACKNOWLEDGMENT}

The authors are pleased to acknowledge the helpful discussions with Mr. H.Ito. They would also like to thank Mr. T.Yamagishi and Mr. M.Hirasaki for forming targets and for evaluating the long term stability of the media, respectively. They express their thanks to Mr. E.Momosaki and Mr. T.Saito for their encouragement.

\section{REFERENCES}

[1] N.Imamura, S.Tanaka, F.Tanaka and Y.Nagano: IEEE Trans. Magn., MAG-21(5), 1607,1985

[2] S.Shimokawato, S.Funada, T.Yamagishi, T.Shimoda: Paper 21B-14, International Symposium on Magneto-Optics, April 20-22, 1987, Kyoto

[3] S.Funada, S.Shimokawato, M.Ishida, T.Shimoda: Paper CG-02, Intermag, April 14-17, 1987, Tokyo (to appear in IEEE Trans. Magn., MAG-23,1987)

[4] M.Kobayashi, M.Asano, K. Kawamura and S.Ohno: Journal of Magnetic Society of Japan, vol.9, 93, 1985 (in Japanese) 